\title{
Neuroendocrine Tumors of the Breast: Single-Center Experience
}

\author{
(1) Bermal Hasbay ${ }^{1}$, (1) Hüseyin Özgür Aytaç², (D) Filiz Aka Bolat ${ }^{1}$ \\ ${ }^{1}$ Department of Pathology, Başkent University Turgut Noyan Hospital, Adana, Turkey \\ ${ }^{2}$ Department of General Surgery, Başkent Universty Faculty of Medicine, Adana, Turkey
}

\begin{abstract}
Objective: Breast carcinomas with neuroendocrine (NE) differentiation are extremely rare. The aim was to discuss breast cancer cases with NE differentiation in the light of World Health Organization 2019 classification and literature information.

Material and Methods: The pathology records of 56 cases diagnosed as neuroendocrine tumor (NET) and/or breast cancers with NE differentiation presenting to a single center between January 2010 and June 2020 were evaluated. The patients were evaluated in terms of age, tumor size, location, histological grade, hormone profiles (ER, PR, HER2), guideline American Joint Committee on Cancer, lymph node status, stage, metastases, progression, survival, radiological features, surgery type and therapy modality.

Results: The age of the patients ranged from 34 to 81 years. Average tumor size was $2.3 \mathrm{~cm}$. Median (range) follow up time was 31.5 (1-73 month). Metastatic lymph nodes were found in 20 cases. In our series, NE differentiation mostly accompanied invasive carcinoma of no special type, less frequently solid papillary carcinoma, and mucinous carcinoma.

Four patients had a history of neoadjuvant chemotherapy. Response to treatment was very poor in all four cases. Synaptophysin and chromogranin were positive in 38 cases. No correlation was found among tumor size, grade, age, lymph node status, and presence of distant metastasis in our series.

Conclusion: Clinical features and morphology may not help to distinguish NET from other subtypes of breast cancer. Therefore, the morphologic findings of a nested or trabecular architecture, nuclear or cytoplasmic features of NE differentiation, mucin production, or solid papillary growth pattern should prompt a pathologist to order NE markers.
\end{abstract}

Keywords: Neuroendocrine, breast, solid papillary, mucinous

Cite this article as: Hasbay B, Aytaç HÖ, Aka Bolat F. Neuroendocrine Tumors of the Breast: Single-Center Experience.

Eur J Breast Health 2022; 18(1): 30-36

\section{Key Points}

- $\quad \mathrm{NE}$ markers should be added when morphologically suspected or in SPC and MC cases to determine the actual rate of NE tumors of the breast.

- As these tumors are rare; diagnosis requires exclusion of metastasis from an extra-mammary site.

\section{Introduction}

Primary breast carcinoma with neuroendocrine (NE) features is a rare subtype of breast cancer. NE differentiation in breast carcinomas was first described by Feyrter and Hartmann in 1963 (1-5). In 1977, Cubilla and Woodruff (6) published the first case series and coined the term "primary carcinoid of the breast" (1-3, 6). Sapino et al. (7) in 2001 proposed the first diagnostic criteria for neuroendocrine tumors (NETs) of the breast, suggesting that tumors with more than $50 \%$ of the expression of NE markers, specifically synaptophysin (SNP) and chromogranin, should be classified as primary NE breast carcinomas $(1,7,8)$. In 2003, the World Health Organization (WHO) divided neuroendocrine carcinomas (NECs) into solid, small cell, and large-cell NECs $(1,2,9)$. The term "NEC of the breast" was revised to "carcinomas with NE features" in the 2012 WHO Classification of Tumours of the Breast (10). In 2012, the WHO classification was revised, and minimum percentage of cells exhibiting positive immunostaining for NE markers was removed $(2-4,10)$. Carcinomas with NE features are subclassified into three groups: well-differentiated NET, poorly differentiated NEC/small-cell carcinoma, and invasive breast carcinoma with NE differentiation (1, 2, 10). 
According to the 2019 WHO classification, most NE neoplasms of the breast presumably represent mixed NETs, with most cases showing a component of classic-type mammary carcinoma. Similarly, the majority of primary small-cell NEC (SCNEC) of the breast show a component of classic-type mammary carcinoma. Therefore, if SCNEC makes up 10\%-90\% of the tumour area, the terminology of mixed invasive carcinoma (NST or other special type) and SCNEC may be used, and the NEC percentage should be reported. Cancers with $<10 \%$ NET pattern should be classified as invasive carcinomanon-spesific type (IC-NST) or other types, with an option to describe the focal specialized NE pattern in the report comment. Cancers with $>90 \%$ NE neoplasm pattern should be classified as NET or NEC (11).

NETs in other sites, such as the lungs and gastrointestinal tract, could easily be recognised by their classical growth patterns (solid, alveolar, ribbons, cords, nested and rosette formation) and cytonuclear features (salt and pepper chromatin distrubition) (2). NET/well-differentiated subgroup and the poorly differentiated/small-cell carcinoma are easy to distinguish because they exhibit NE features. Invasive breast carcinoma eith NE differentiation is usually overlooked because they lack the typical morphological features of NE tumors. Recognation of this group by pathologists would help determine the actual frequency of this tumor and its effect on prognosis.

As well as primary NETs of the breast, metastatic NE tumors have also been reported. Clinical and radiological examinations are essential to differentiate a primary invasive breast carcinoma with NE features from a metastatic NE carcinoma. The presence of ductal carcinoma in situ (DCIS) components and extensive positive immunstaining for estrogen receptor/progesterone receptor (ER/PR) within the tumor suggest the primary origin to be the breast $(4,10,12)$.

The most common form of NE breast tumor-solid papillary carcinoma (SPC) and mucinous carcinoma (MC) is a suitable example of diagnostic and conceptual challenges with NET $(8,13,14)$. However, SPC is a distinctive clinico-pathological entity that often expresses NE markers.

The prognostic relevance of the NE differentiation of breast tumors is still debated. The present study aimed to evaluate breast carcinomas showing NE differentiation in terms of histopathological features, hormone receptor status, radiological features, and treatment modalities.

\section{Material and Methods}

The pathology archive of our hospital between January 2010 and June 2020 were evaluated and found cases diagnosed as NETs and/ or breast cancers with NE differentiation were identified. Clinical follow-up was obtained from the electronic data system and record archive of our center. A 10-year electronic data search was performed with the laboratory information system using the keywords "breast" and "neuroendocrine tumor/NE differentiation" for diagnosis. In addition, $\mathrm{MC}$ cases without NE differentiation were compored with MC cases showing NE differentiation. SNP, chromogranin, ER, PR, human epidermal growth factor receptor 2 (HER2), and $\mathrm{Ki}-67$ were studied in the cases with histopathological NETs. If NE differentiation areas were suspected in primary breast tumor, SNP and chromogranin were studied first. When both were negative, neuron-specific enolase (NSE) and CD56 were added. When one or two of them were found to be positive by $10 \%$ or more with immunohistochemistry, invasive breast carcinoma (mucinous, solid,
IC-NST, lobular), showing NE differentiation was diagnosed. NET or NEC was diagnosed when $90 \%$ or more positivity was observed to accompany histological features.

For each cases; age, location, tumor size, histologic grade, the presence of associated DCIS, lymphovascular invasion, perineural invasion, microcalcification, nodal metastasis, hormone receptors, tumor type, follow-up duration and outcome (dead or alive, presence or absence of local recurrence or metastasis), and treatment modalities were also documented.

In accordance with the American Society of Clinical Oncology-College of American Pathologists (ASC0-CAP) guidelines, the tumor was defined as positive for ER and PR if positive nuclear staining was noted for $\geq 1 \%$ of the invasive tumor cells (15). HER2 immunhistochemical expression was scored in accordance with ASC0-CAP guidelines (16): 0 , no staining or weak-moderate incomplete stainig in $\leq 10 \%$ of cells; 1 , weak and incomplete staining in $>10 \%$ of cells; 2 , weak-moderate staining in $>10 \%$ of cells or strong staining in less than $10 \%$ of cells; and 3 , strong complete membranous staining in $10 \%$ of cells. Cases suspicious for HER2 overexpression (Score 2) underwent further fluorescence in situ hybridization (FISH) analysis. When the ratio of Cerb2/chromosome 17 was $<2$ and $\geq 2$, it was accepted as negative and positive for gene amplification, respectively.

Statistical analysis was performed using the SPSS software, version 17.0 (IBM, Inc., Chicago, IL, USA). The normality of each continuous variable was checked by Shapiro-Wilk tests and by histograms. All numerical data were expressed as median values (minimum-maximum) or as proportions. The Kaplan-Meier method was used for the survival analysis.

Ethics committee approval was obtained from Başkent University Medicine and Health Sciences Research Board (decision no: KA21/399, date: 08.10.2021).

Written consent was not obtained from the patients since the study was designed retrospectively and needed no consent.

\section{Results}

Results showed that 59 patients had undergone biopsy, including 56 primary breast NETs. Three of the 59 tumor cases were excluded because of metastases to the breast. Thus, 56 patients were included in the study (Table 1). Microcalcifications were observed in nine (16.1\%) of the cases. SNP (Figure 1) was positive in 50 (89.3\%), and negative in six $(10.7 \%)$ cases, whereas chromogranin (Figure 2 ) showed positive staining in $41(73.2 \%)$, and negative staining in $17(30.4 \%)$ cases. SNP and chromogranin were both positive in 38 (67.9\%) cases. NSE was positive in eight $(14.3 \%)$ cases. The mean Ki-67 proliferation index was $14.9 \%$ (range: $2-70$ ). Regarding the molecular subtypes of NET, 34 (78.6\%) were ER +/Her2- (Luminal A), and 12 (21.4\%) were ER+/HER2+ / - and Ki67 > 14\% (luminal B).

The mean age at diagnosis was 57.2 years, with a median of 60 years (34-81). Fifteen cases were premenopausal (age $<50,26.8 \%$ ), and 41 cases were postmenopausal (age $>50,73.2 \%$ ). Average tumor size was $2.3 \mathrm{~cm}(0.3-7 \mathrm{~cm})$. In addition, $26(46.4 \%)$ of the cases were located in the right breast and $30(53.6 \%)$ were in the left breast. Multifocality was noted in six of the 56 cases $(10.7 \%)$. The patients mostly presented because of a complaint of a palpable mass. In addition, $53(96.4 \%)$ of the cases were women and $3(5.4 \%)$ were men. Of the 56 cases, two 
(3.6\%) were dead, 54 (96.4\%) were alive. Bilateral breast carcinoma was present in three of the cases. Morever, 10 (17.9\%) patients had a family history of breast cancer. Median follow-up time was 31.5 (1-73) months. The estimated mean life expectancy of all patients was $41 \pm 18.9$ months.

Twenty-one patients underwent mastectomy with sentinel lymph node biopsy (SLNB), 31 patients underwent breast conserving surgery with SLNB. Two cases were those evaluated with consultation blocks. Another two cases were diagnosed with core biopsies. Metastatic lymph nodes were observed in $20(38.5 \%)$ of 52 cases with lymph node sampling, whereas lymph nodes were reactive in the remaining 32 cases. In terms of $\mathrm{N}$ staging, 32 cases were pN0 (57.2\%), 15 cases were $\mathrm{pN} 1$ (26.8\%), one case was $\mathrm{pN} 2(1.8 \%)$, four cases $\mathrm{pN} 3(7.1 \%)$ and four cases $\mathrm{pNx}$. The $\mathrm{pNx}$ stage consisted of two consultation cases, and the two patients were diagnosed with core biopsy.

In accordance with the Modified Bloom and Richardson score, five cases were Grade 1 (8.9\%), 23 cases Grade 2 (41.1\%), and 28 cases Grade 3 (50\%). When evaluated in terms of pT: one $(1.8 \%)$ case was pT in situ, $21(37.5 \%)$ cases pT1, 26 (46.4\%) cases pT2, three (5.4\%) cases pT3, one (1.8\%) case pT4 and four cases $(7.1 \%)$ pTx. The pTx stage consisted of two consultation cases, and two patients were diagnosed with core biopsy. Our archive records contained 81 MC cases (33 pure MCs and 48 MCs with mixed carcinomas) without $\mathrm{NE}$ differentiation. We did not find any significant difference between these two groups in terms of $\mathrm{pT}(\mathrm{p}=0.081), \mathrm{pN}$ $(\mathrm{p}=0.118)$, DCIS $(\mathrm{p}=0.719)$, grade $(\mathrm{p}=0.595)$, hormone receptor positivity $(\mathrm{p}=0.414)$, age $(\mathrm{p}=0.022)$, follow-up time $(\mathrm{p}=0.043)$ and Ki-67 score $(\mathrm{p}=0.417)$.

Radiotherapy (RT) only was performed in seven (12.5\%) patients and chemotherapy (CT) only was also performed in seven patients. CT and RT were performed in 28 (50\%) patients. Eight (7.1\%) patients received hormone therapy alone. Tamoxifen was added in the treatment of ER positive patients, and Trastuzumab in HER2 positive patients. Of the 56 patients, six $(10.7 \%)$ were lost to followup, and the follow-up period for the remaining 50 patients ranged from 1 to 73 months (31.5). Among these 56 patients, one patient

Table 1. Original diagnosis of 56 cases showing neuroendocrine features

Diagnosis and number of cases with NE differentiation n (\%)

\section{IC-NST}

Solid papillary carcinoma with invasion

With invasive mucinous carcinoma

With IC-NST

Mixed IC-NST + mucinous carcinoma

Mucinous carcinoma

Solid papillary carcinoma

Mixed IC-NST + mucinous carcinoma

Mixed IC-NST + lobular carcinoma

IC-NST + Poorly differentiation NET

Totally

$56(100 \%)$

NE: Neuroendocrine; IC-NST: Invasive carcinoma-carcinoma of no special type; n: Number died of the disease after 24 months. The other case who was dead was a patient diagnosed by core biopsy and was not followed up. Clinicopathological characteristics of 56 patients are summarized in Table 2.

Four (7.1\%) patients had a history of neoadjuvant CT. Two of these were IC-NST with NE differentiation, and two were invasive MC with NE differentiation. Response to treatment was very poor in all four cases. Four of the patients had a second primary carcinoma accompanying breast carcinoma. Two of them were non-Hodgkin's lymphoma, one was oncocytoma and one was endometrium carcinoma.

\section{Discussion and Conclusion}

Primary NE carcinoma of the breast includes a heterogeneous group of tumors with different biological behavior and prognosis (3). The incidence has been reported to range from $<1 \%-5 \%$ of breast cancers. In contrast, some authors reported NE differentiation in up to $20 \%$ of breast carcinomas (3). However, the exact incidence of this disesase is difficult to assess because immunohistochemical NE markers are not routinely used in breast tumors $(3,10)$.

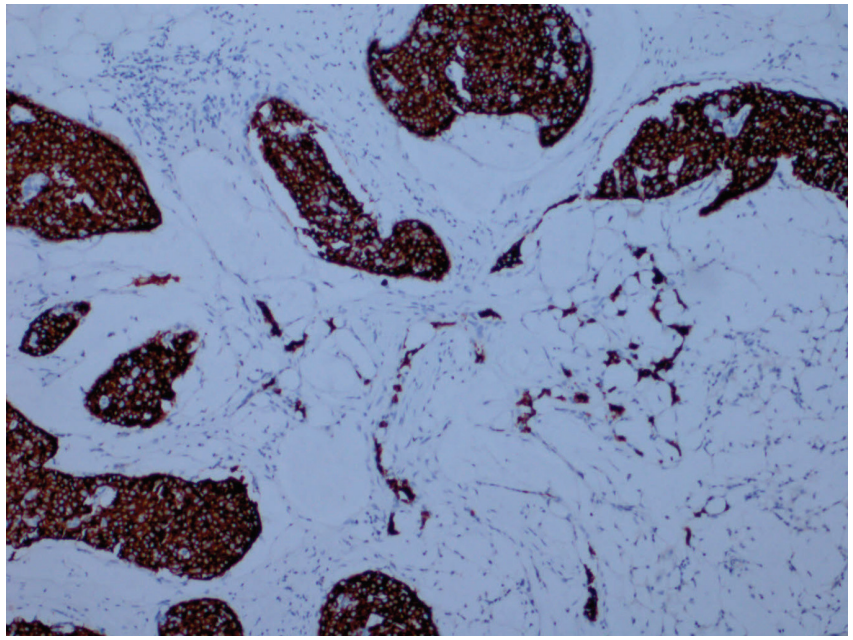

Figure 1. Immunohistochemically, SNP positivity in tumor cells $(\mathrm{IHK} \times 200)$

SNP: Synaptophysin; IHK: Immunohistochemistry

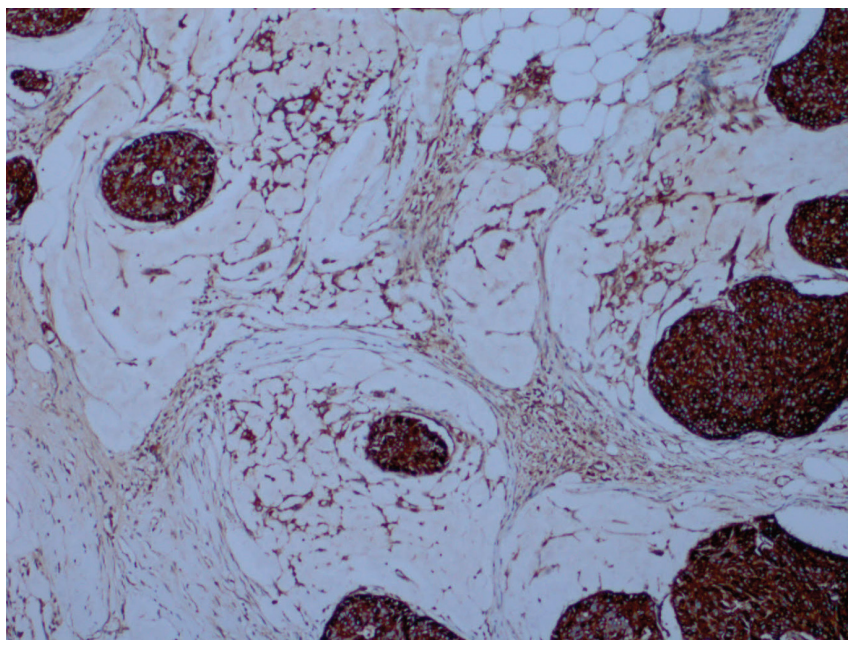

Figure 2. Immunohistochemically, chromogranin positivity in tumor cells $($ IHK $\times 200)$

IHK: Immunohistochemistry 
Table 2. Clinico-pathological characteristics of 56 patients

n

(\%)

\section{Age group}

Mean age

Median (range) age

Age $<50$

Age $>50$

Tumor location

Right

Left

DCIS

Present

Absent

73.2

Estrogen receptor (ER)

Positive

56

100

Negative

0

Progesterone receptor (PR)

Positive

8

85.7

Negative

HER2/neu

Positive

Negative

8

48

14.3

85.7

\section{Tumor size}

Mean size

Median size

$2.3 \mathrm{~cm}(0.3-7)$

$1.8 \mathrm{~cm}$

\section{Histologic grade}

G1

G2

G3

PN

pNO

$\mathrm{pN} 1$

pN2

pN3

$\mathrm{pNx}$

LN

Present

Absent

\section{Table 2. Continued}

n

PT

PT in situ

pT1

PT2

pT3

PT4

Unknown

Metastasis

Bone

Liver

No metastasis

LVI

Present

Absent

PNI

Present

Absent

Surgery

$M$ and SLND

SM and SLND

Unknown

Systemic therapy

$\mathrm{CT}+\mathrm{RT}$

CT

RT

TMX

Unknown

Final status

Alive

Dead

SLND: Sentinel lymph node dissection; M: Mastectomy; SM: Segmented mastectomy; CT: Chemotherapy; RT: Radiotherapy; PT: Pathologic tumor stage; pN: Pathologic nodal stage; DCIS: Ductal carcinoma in situ; IC-NST: Invasive carcinoma-carcinoma of no special type; TMX: Tamoxifen; LVI: Lymphovascular invasion; PNI: Perineural invasion; LN: Lobular neoplasia; n: Number 
NETs of the breast occur predominantly in postmenopausal women during the sixth to seventh decade of life, although rare cases have been reported in males $(1,3)$. In this study, most of the cases $(73.6 \%)$ were in the postmenopausal period, with a median (range) age 60 (34-81) years. This situation is similar to the literature. Three of our cases were male. The tumor size of NETs of the breast ranges from 0.8 to 13.5 $\mathrm{cm}$ with a mean of $2.7 \mathrm{~cm}(1,17)$. Similarly, average tumor size was $2.3 \mathrm{~cm}(0.3-7 \mathrm{~cm})$ in our series. Tumors may be grossly infiltrative or expansile, and those with mucin production are soft and gelatinous $(1,10)$. Microcalcification was identified in a small number of cases in our series $(n=9,16 \%)$ which is consistent with that reported in the literature (10\% and $25 \%)$.

Two main theories exist on the histogenesis of primary NETs of the breast. The first theory is that these tumors evolve from neoplastic transformation of native NE cells. The second and more accepted theory is that NE differentiation arises from divergent differentiation of neoplastic stem cells into epithelial and endocrine cell lines during early carcinogenesis. This theory is supported by the lack of benign NETs of the breast and evidence that NE cells are clonally related to malignant epithelial cells $(1,3)$.

$\mathrm{NE}$ differentiation is frequently found in $\mathrm{MC}$, particularly the hypercellular variant, and SPC (1). However, the expression of NE markers is not unique to $\mathrm{MC}$ of the breast (18). This phenomenon has been described in other breast carcinomas, including infiltrating lobular carcinoma, IC-NST (18). Invasive lobular carcinoma, particularly the alveolar variant, can also demonsrate NE differentiation (19). In our series, mostly IC-NST, less frequently SPC and MC were observed. These histopathological subtypes with similar frequencies were reported in previous studies (2). In our series, NE differentiation areas were found in 44 IC-NST carcinoma cases. Meanwhile 32 of these cases were pure IC-NST, and 11 had mixed breast carcinoma (five cases SPC + IC-NST, two cases MC + IC-NST, one case invasive lobular carcinoma + IC-NST, one case SPC + MC + IC -NST (Figure 3), and 1 case IC-NST + poorly differentiation / small-cell carcinoma NET).

MC is histologically characterized by nests of tumor cells floating in mucin lakes with fine fibrovascular septae (10). NE differentiation

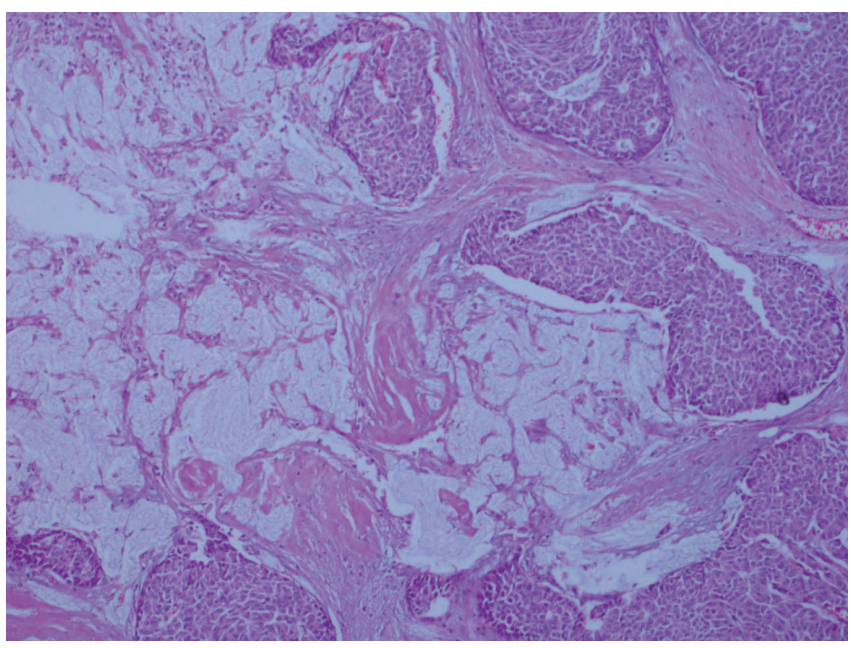

Figure 3. Mucinous carcinoma developed on the basis of solid papillary carcinoma, showing neuroendocrine differentiation $(H \& E \times 200)$ is more frequently observed with the hypercellular variant of $\mathrm{MC}$, characterized by large clusters of tumor cells (1). In our series, NE differentiation was observed in 15 cases with MC. The significance of NE differentiation in $\mathrm{MC}$ has been controversial. Some authors reported a difference in the age and prognosis of patients, whereas others found no such difference (18). Our archive records contained $81 \mathrm{MC}$ cases without NE differentiation. No significant difference was found between these MC cases and the NET/NE differentiation group in terms of classification of $\mathrm{pT}$ and $\mathrm{pN}$ rate of DCIS, grade, hormone receptor positivity, age, follow-up time and $\mathrm{Ki}-67$ score.

SPC is a rare form of breast carcinoma composed of large circumscribed nests of small monotonous polygonal to spindled cells, fine fibrovascular cores, and a round to elongated nucleus, plus finely granular eosinophilic cytoplasm $(1,13,14)$. NE differentiation is present in up to $50 \%$ of cases. Our archive contained 17 cases of SPC, 15 of which had NE differentiation areas. Two of the SPC cases with NE differentiation in our series were pure SPC, and 13 cases with invasive breast carcinoma (five cases MC, five cases IC-NST, three cases IC-NST + MC) developed on an SPC backround. SPC usually arises in the seventh or eighth decade and has a better prognosis than other breast cancers $(13,14)$. Concordantly, the mean age of patients with SPC in our series was 62.

DCIS can also display NE differentiation, especially in solid-type DCIS. Endocrine DCIS is often of low nuclear grade, with eccentric nuclei and open chromatin $(1,20)$. In our series, 41 (73.2\%) patients had DCIS, with the most frequent patterns being solid, cribriform, comedo, NE, and papillary. SNP was positive in 37 patients, and chromogranin was positive in 27 patients.

Although morphological features may suggest NE differentiation, the diagnosis of NET requires expression of NE markers. The most sensitive and spesific immunohistochemical markers are SNP and chromogranin A $(1,20)$. NSE and CD56 may show positivity but are less sensitive and specific (1). Ki-67 is a prognostic indicator of NETs (21). In our series, SNP was positive in 50 cases and negative in six cases, whereas chromogranin was positive in 41 cases and negative in 17 cases. SNP and chromogranin were positive in 38 cases. NSE was positive in eight cases.

The series reported in the literature were mostly of the ER+/Her2 luminal A molecular subtype. (1-3, 8). Studies have shown that NETs are more likely to be ER and PR positive than IC-NST (1). Wei et al. (22) demonstrated that $95 \%$ of NETs are ER positive, $80 \%$ are PR positive and $91 \%$ are HER 2 negative. In our series, all cases were ER positive, and 85\% were PR positive while 14\% HER2 amplified. Regarding the molecular subtypes of NETs, more than three quarters were ER +/Her2 - (Luminal A), and while a fifth were ER+/HER2+ I- and $\mathrm{Ki}-67>14 \%$ (luminal B). Six of the Her2 positive cases were IC-NST, one was IC-NST + invasive lobular carcinoma, and the other was invasive $\mathrm{MC}$.

The differential diagnosis of NET of the breast is broad and includes benign and malignant entities. The most important differential diagnosis is metastatic NET from an extramammary site, as well as lymphoma and malignant melanoma (1). Metastatic NETs account for $1 \%-2 \%$ of metastases to the breast. Few cases of metastatic $\mathrm{NE}$ carcinoma to breast were noted in the review of literature. The majority of these were from the small intestine and the pancreas (23). The distinction of primary from metastatic NET is critical to avoid 
misdiagnosis and unnecessary surgical and medical therapy in the latter (1). Approximately $68 \%$ of primary NETs are associated with DCIS, which is the most convincing evidence of a primary breast tumor $(1,2)$. A panel of immunohistochemical stains can prove useful in distinguishing these two entities. As both primary and metastatic tumors show NE differention, neither NE markers nor ER and PR, which can also show positivity in metastasis, are useful in distinguishing the diagnosis (24). The most specific markers for a breast primary tumor are GATA3, mammaglobin, and GCDFP15, for which secondary tumors are consistently negative $(2,24)$. TTF 1 shows positivity in approximately $70 \%$ of metastases from the lung and CDX2 shows positivity in $100 \%$ of metastases from the gastrointestinal tract $(2,24)$. TTF1 may be strongly positive in poorly differentiated NETs of the breast (1). Therefore, especially when ruling out lung NET metastasis, attention should be paid to hormone receptors in breast tumors, GATA3 and GCDFP15 positivity, and the presence of DCIS. Moreover, obtaining detailed past medical history of patients is important because those with known history of carcinoid tumors may present with metastatic lesions many years after their initial diagnosis.

A spesific guideline for the grading, staging, or treatment of primary NETs of the breast is lacking (10). Similar to conventional breast cancers, NETs of the breast must be staged and treated (22). Surgical management is based on tumor location and stage as with conventional breast cancers (22). Well-differentiated NET and invasive breast carcinoma with NE differentiation receive cytotoxic therapy similar to conventional breast cancer, and those with poorly differentiated NETs receive cytotoxic therapy with protocols similar to that of pulmonary small-cell carcinoma. The use of hormone therapy should be based on receptor status.

Tumor size and nodal metastases are the main prognostic factors for evaluating risk of relapse for NET of the breast, as for other types of breast cancers (3). NET of the breast can metastasize to multiple sites several years after the treatment for primary tumor. Therefore, a long-term follow-up is advisable. Metastatic sites include liver, bones, lungs, pancreas and brain (3). In our series, two cases had metastasized to the bone and one case to the liver. Although no consensus has been reached on the clinical or prognostic significance of this entity, many large studies that used updated criteria suggest poor prognosis. In our series, no statistically significant relation was observed in terms of tumor size, nodal metastasis, grade, survival, age, and prognostic terms.

Breast carcinoma with NE differentiation is a heterogeneous disease composed of many different subtypes with varying clinical characteristics. As these tumors are rare, diagnosis requires exclusion of metastasis from an extra-mammary site. Clinical features and morphology may not be helpful to distinguish NET from other subtypes of breast cancer. Therefore, the morphologic findings of a nested or trabecular architecture, nuclear or cytoplasmic features of NE differentiation, mucin production, or a solid papillary growth pattern should prompt a pathologist to order markers specific SNP and chromogranin. Similar regimens to conventional breast carcinoma are used in terms of treatment; but neoadjuvant CT response was poor in the small number of cases in our series. However, larger series are needed to predict the need for different treatment protocols or to decide on prognosis. As NE markers are not used routinely, the exact frequency of this tumor type remains unknown. Therefore, NE markers should be added when morphologically suspected, or in SPC and MC cases to determine the true rate of NE tumors of the breast.

Ethics Committee Approval: This study was approved by Başkent University Medicine and Health Sciences Research Board (decision no: KA21/399, date: 08.10.2021).

Informed Consent: Retrospective archive research.

Peer-review: Externally peer-reviewed.

\section{Authorship Contributions}

Conception: B.H., F.A.B.; Design: B.H.; Supervision: H.Ö.A., F.A.B.; Materials: B.H., H.Ö.A., F.A.B.; Analysis and/or Interpretation: B.H., F.A.B.; Writing: B.H., H.Ö.A.

Conflict of Interest: No conflict of interest declared by the authors.

Financial Disclosure: The authors declare that this study received no financial disclosure.

\section{References}

1. Rosen LE. Neuroendocrine tumors of the breast. Arch Pathol Lab Med 2017; 141: 1577-1581. (PMID: 29072945) [Crossref]

2. Talu CK, Leblebici C, Ozturk TK, Hacihasanoglu E, Koca SB, Gucin Z. Primary breast carcinomas with neuroendocrine features: clinicopathological features and analysis of tumor growth patterns in 36 cases. Ann Diagn Pathol 2018; 34: 122-130. (PMID: 29661717) [Crossref]

3. Inno A, Bogina G, Turazza M, Bortesi L, Durantı S, Massocco GZ, et al. Neuroendocrine carcinoma of the breast: current evidence and future perspectives. Oncologist 2016; 21: 28-32. (PMID: 26659223) [Crossref]

4. Talu CK, Savli TC, Huq GE, Leblebici C. Histopathological and clinical differences between primary breast carcinomas with neuroendocrine features and primary breast carcinomas mimicking neuroendocrine features. J Surg Pathol 2019; 27: 744-752. (PMID: 31195855) [Crossref]

5. Lavigne M, Menet E, Tille JC, Lae M, Fuhrmann L, Bonneau C, et al. Comprehensive clinical and molecular analyses of neuroendocrine carcinomas of the breast. Modern Pathol 2018; 31: 68-82. (PMID: 28884749) [Crossref]

6. Cubilla AL, Woodruff JM. Primary carcinoid tumour of the breast: a report of eight patients. Am J Surg Pathol 1977; 4: 283-292. [Crossref]

7. Sapino A, Papotti M, Righi L, Cassoni P, Chiusa L, Bussolati G. Clinical significance of neuroendocrine carcinoma of the breast. Ann Oncol 2001; 12(Suppl 2): 115-117. (PMID: 11762336) [Crossref]

8. Visscher DW, Yasir S. Neuroendocrine tumors of the breast. Endocr Pathol 2017; 28: 121-127. (PMID: 28389994) [Crossref]

9. Ellis IO, Schnitt SJ, Sastre-Garau X. Invasive breast carcinoma. In: Tavassoli FA, Devilee P, editors. Pathology and genetics of tumours of the breast and female genital organs. 3rd ed. Lyon, France: IARC Press; 2003. p. 32-34. [Crossref]

10. Bussolati G, Badve S. Carcinomas with neuroendocrine features. In: Lakhani SR, Ellis IO, Schnitt SJ, Tan PH, van der Vijver MJ. WHO Classification of Tumours of the Breast. Lyon, France: IARC Press; 2012. p. 62-63. [Crossref]

11. Allison KH, Brogi E, Ellis IO, Fox SB, Morris EA, Sahin A. The WHO Classification of Tumours Breast Tumours. 5th ed. USA: IARC; 2019. p. 155-161. [Crossref]

12. Gagno S, D'Andrea MR, Mansutti M, Zanusso C, Puglisi F, Dreussi E, et al. A new genetic risk score to predict the outcome of locally advanced or 
metastatic breast cancer patients treated with first-line exemastane: results from a prospective study. Clin Breast Cancer 2019; 19: 137-145. (PMID: 30584056) [Crossref]

13. Okubo Y, Okubo T, Okubo Y, Ishiwatari T. Neuroendocrine Differentiation in Breast Cancer: Clinicopathological Significance of Bcl-2 Positive Solid Papillary Carcinoma. Case Rep Med 2016; 2016: 9501410. (PMID: 28105053) [Crossref] doi: 10.1155/2016/9501410. [Crossref]

14. Guo S, Wang Y, Rohr J, Fan C, Li Q, Li X, et al. Solid papillary carcinoma of the breast: A special entity needs to be distinguished from conventional invasive carcinoma avoiding over-treatment. Breast 2016; 26: 67-72. (PMID: 27017244) [Crossref]

15. Hammond ME, Hayes DF, Dowsett M, Allred DC, Hagerty KL, Badve $S$, et al. American Society of Clinical Oncology/College of American Pathologists guideline recommendations for immunohistochemical testing of estrogen and progesterone receptors in breast cancer. J Clin Oncol 2010; 28: 2784-2795. (PMID: 20404251) [Crossref]

16. Wolff AC, Hammond ME, Hicks DG, Dowsett M, McShane LM, Allison KH, et al. American Society of Clinical Oncology; College of American Pathologists. Recommendations for human epidermal growth factor receptor 2 testing in breast cancer: American Society of Clinical Oncology /Colloge of American Pathologists clinical practice guideline update. Arch Pathol Lab Med 2014; 138: 241-256. (PMID: 24099077) [Crossref]

17. Adams RW, Dyson P, Barthelmes L. Neuroendocrine breast tumours: breast cancer or neuroendocrine cancer presenting in the breast? Breast 2014; 23: 120-127. (PMID: 24342375) [Crossref]

18. Tse G, Ma T, Chu W, Lam W, Poon C, Chan WC. Neuroendocrine differentiation in pure type mammary mucinous carcinoma is associated with favorable histologic and immunohistochemical parameters. Mod Pathol 2004: 17: 568-572. (PMID: 15001999) [Crossref]

19. Tang F, Wei B, Tian Z, Gilcrease MZ, Huo L, Albarracin CT, et al. Invasive mammary carcinoma with neuroendocrine differentiation: histological features and diagnostic challenges. Histopathology 2011; 59: 106-115. (PMID: 21668471) [Crossref]

20. Hoda SA, Brogi E, Koermer FC, Rosen PP. Rosen's breast pathology. 4th ed. 2014: 667-688. [Crossref]

21. Moyana TN, Xiang J, Senthilselvan A, Kulaga A. The spectrum of neuroendocrine differentiation among gastrointestinal carcinoids: importance of histologic grading, MIB-1, p53, and bcl-2 immunoreactivity. Arch Pathol Lab Med 2000; 124:570-576. (PMID: 10747315) [Crossref]

22. Wei B, Ding T, Xing Y, Wei W, Tian Z, Tang F, et al. Invasive neuroendocrine of the breast: a distinct subtype of aggressive mammary carcinoma. Cancer 2010; 116: 4463-4473. (PMID: 20572042) [Crossref]

23. Lee S, Levine P, Heller SL, Hernandez O, Mercado CL, Chhor CM. Metastatic carcinoid tumor to the breast: report of two cases and review of the literature. Clin Imaging 2017; 42: 88-92. (PMID: 27907837) [Crossref]

24. Mohanty SK, Kim SA, Delair DF, Bose S, Laury AR, Chopra S, et al. Comparison of metastatic neuroendocrine neoplasms to the breast and primary invasive mammary carcinomas with neuroendocrine differentiation. Mod Pathol 2016; 29: 788-798. (PMID: 27125358) [Crossref] 